\title{
Sistemas ultrasónicos para la irrigación del sistema de conductos radiculares
}

\section{Ultrasonic devices for root canal system irrigation}

\author{
García Delgado A*, Martín-González J*, Castellanos-Cosano L*, Martín Jiménez M*, \\ Segura-Egea JJ*
}

\section{RESUMEN}

La eliminación de los restos de tejido pulpar vital y necrótico y de los microorganismos del sistema de conductos radiculares, es esencial para el éxito en endodoncia. La desinfección del canal radicular mediante la irrigación e instrumentación es el factor más importante en la prevención y tratamiento de la periodontitis apical. Al ser imposible con la instrumentación llegar a todas las áreas del sistema de conductos, la irrigación cobra especial importancia. Por este motivo, en la última década se han desarrollado una serie de sistemas de dispensación y agitación de irrigantes, tales como los ultrasonidos. En concreto, en este artículo de revisión se evalúa la información disponible de los diez últimos años sobre la efectividad de los sistemas ultrasónicos para eliminar bacterias, tejido pulpar, restos de dentina y barrillo dentinario, la capacidad de estos dispositivos para hacer que el irrigante penetre en el sistema de conductos radiculares y la seguridad en su uso. Los resultados de la revisión muestran que los sistemas ultrasónicos son más eficaces en el desbridamiento químico, biológico y físico del sistema de conductos radiculares que los sistemas de irrigación convencional, a la vez que son seguros.

Palabras clave: Ultrasonidos, irrigación ultrasónica, PUI, CUI, endodoncia.

\section{SUMMARY}

Removal of vital and necrotic remnants of pulp tissues and microorganisms from the root canal system is essential for endodontic success. Disinfection of the root canal by irrigation and instrumentation are the most important factors in the prevention and treatment of apical periodontitis. As it is impossible for the instruments to reach all the areas of the root canal system, the irrigation has gained special importance. Due to this fact, technological advances during the last decade have brought new delivery and agitation devices, such as ultrasonic devices. Particularly, this review article assesses the available information from the last ten years about the effectiveness of ultrasonic devices to remove bacteria, pulp tissue, dentin debris and smear layer, and the ability of these devices to make the irrigant solution penetrate into the root canal system and the safety of its use. Nowadays scientific literature reveals that ultrasonic devices are more effective on chemical, biological and physical debridement of the root canal system than conventional technique and being safety devices at the same time.

Key words: Ultrasound, ultrasonic irrigation, PUI, CUI, endodontics.

Fecha de recepción: 20 de mayo de 2013.

Aceptado para publicación: 10 de julio de 2013.

* Grupo de Investigación en Endodoncia CTS-941, Departamento de Estomatología. Universidad de Sevilla. Sevilla, España.

García Delgado A, Martín-González J, Castellanos Cosano L, Martín Jiménez M, Sánchez Domínguez B, SeguraEgea JJ. Sistemas ultrasónicos para la irrigación del sistema de conductos radiculares. Av. Odontoestomatol 2014; 30 (2): 79-95. 


\section{INTRODUCCIÓN}

La limpieza y desinfección de todas las áreas del conducto radicular mediante soluciones irrigadoras se considera esencial para el éxito del tratamiento endodóncico (1). Numerosos estudios han demostrado que durante la preparación mecánica, quedan muchas zonas del conducto que ni siquiera son tocadas por los instrumentos (2), actuando únicamente sobre el cuerpo central del conducto (3). Además de la complejidad anatómica del propio diente, se suma el problema que supone el vapor lock. Debido a que las raíces de los dientes están rodeadas por el ligamento periodontal y el hueso, que "cierran" el foramen apical, el sistema de conductos se comporta como una cavidad de extremo cerrado, produciéndose un atrapamiento de aire cuando se introduce la solución irrigadora. Este efecto hace que, en la mayoría de los casos, el irrigante no alcance el tercio apical del conducto $(4,5)$.

Tomando conciencia de lo importante que son estos factores, se han diseñado un gran número de dispositivos para la limpieza y desinfección del sistema de conductos radiculares, en busca de procedimientos de administración del irrigante más efectivos (6), y de sistemas de agitación del irrigante que faciliten que éste pueda alcanzar las zonas de difícil acceso, donde los instrumentos manuales y rotatorios no pueden llegar $(7,8)$.

Entre estos nuevos dispositivos se encuentran los sistemas ultrasónicos. Se han descrito tres técnicas de irrigación ultrasónica en la literatura. La primera es la instrumentación ultrasónica (ultrasonic instrumentation, UI) en la que se combina la instrumentación y la irrigación ultrasónica simultáneas. Debido a que se producen perforaciones y preparaciones irregulares de forma frecuente, los sistemas UI no son empleados como alternativa a la instrumentación (7). La segunda técnica, denominada irrigación pasiva ultrasónica (passive ultrasonic irrigation, PUI), opera sin instrumentación simultánea, dispensándose primero la solución irrigadora en el interior del conducto y, a continuación, se la agita y activa con ultrasonidos. Una tercera forma de utilizar la irrigación ultrasónica es la irrigación continua (continuous ultrasonic irrigation, CUI). En este régimen de irrigación, el irrigante se dispensa de forma continua mientras se agita. Ambos métodos, tanto PUI como CUI, han mostrado ser eficaces en la eliminación de detritus del conducto (9).

\section{OBJETIVOS}

El objetivo de este trabajo es revisar el estado del conocimiento científico respecto a los sistemas de activación por ultrasonidos de las soluciones irrigadoras en endodoncia, analizando la bibliografía existente en cuanto a cuatro aspectos principales:

1. La eficacia de los sistemas ultrasónicos para erradicar las bacterias del sistema de conductos radiculares.

2. La efectividad de los sistemas de activación ultrasónica del irrigante en la eliminación de los restos pulpares y dentinarios.

3. La capacidad de los sistemas ultrasónicos para eliminar el barrillo dentinario.

4. La validez de los sistemas de activación ultrasónica para conseguir que el irrigante penetre en el conducto principal a la longitud de trabajo, en los conductos laterales y en los túbulos dentinarios y, todo ello, sin provocar la extrusión de la solución irrigadora por el foramen apical hacia el espacio periapical.

\section{MATERIAL Y MÉTODOS}

El material científico se obtuvo de las bases de datos PubMed, MEDLINE y Scopus, ofrecidas por el portal web de la Biblioteca de Centros de la Salud de la Universidad de Sevilla. Para la realización de la búsqueda, se utilizaron las siguientes palabras clave: "ultrasonic irrigation AND (ultrasound $O R$ endodontics OR smear layer). Se localizaron los artículos publicados desde enero del año 2000 hasta abril del año 2013, todos ellos en inglés.

Los criterios de selección de los artículos fueron los siguientes:

- Tipo de artículo: Se incluyeron estudios ex vivo, in vivo y también in vitro, debido a que los sistemas ultrasónicos tal y como se conocen hoy en día son algo muy novedoso y la bibliografía existente al respecto es muy limitada.

- Se tomaron aquellos artículos en los que se comparaban los sistemas ultrasónicos (tanto irrigación pasiva como continua) con otros sistemas (manuales y/o mecanizados) y en los que se valoraban uno o más de los cuatro aspectos nombrados en los objetivos.

- Se excluyeron aquellos artículos que evaluaban la capacidad para eliminar el hidróxido de calcio, 
los que evaluaban aspectos dentro de un único sistema ultrasónico (ej.: Tipos de puntas ultrasónicas) y los que no utilizaban irrigantes convencionales (diferentes al hipoclorito sódico, clorhexidina, EDTA, EDTAC...).

De los 68 artículos encontrados, se seleccionaron 43 que fueron los que se ajustaron a los criterios de selección.

\section{RESULTADOS}

Los 43 artículos que cumplían los criterios de inclusión se han agrupado según el tipo de estudio (in vitro, ex vivo e in vivo) y los aspectos tratados en cada uno de ellos, tal como se muestra en la tabla 1.

\section{Capacidad de erradicación de bacterias}

En 2003, Weber et al. (10) evaluaron el efecto antimicrobiano residual de la activación pasiva ultrasónica de la clorhexidina al $2 \%$ y del hipoclorito sódico al 5,25\%. Se utilizaron 94 dientes recién extraídos con raíces que tenían un único conducto. Se crearon 5 grupos: El grupo 1 consistía en 21 conductos irrigados con CLX al 2\%; El grupo 2 consistía en 21 conductos irrigados con CLX al $2 \%$ más 1 minuto de activación con PUI; El grupo 3 consistía en 21 conductos con $\mathrm{NaOCl}$ al 5,25\%; El grupo 4 consistía en 21 conductos irrigados con $\mathrm{NaOCl}$ seguido de $1 \mathrm{mi}$ nuto de activación con PUI, y el grupo 5 (grupo control) consistía en 10 canales con solución salina tamponada con fosfato (PBS). Los mejores resultados de actividad residual antibacteriana se obtuvieron para el grupo 2 (CLX seguido de 1 min. de PUI), y los peores para el grupo control. Todos los resultados fueron estadísticamente significativos ( $p<0,001)$.

En el mismo año, Spoleti et al. (11) evaluaron la influencia de la activación por PUI sobre la desinfección de los canales radiculares. Se tomaron 60 dientes y se dividieron en 3 grupos principales:

- Grupo A: Incisivos superiores.

- Grupo B: Caninos superiores.

- Grupo C: Raíces distovestibular de primeros molares superiores.

Y, cada uno de ellos, subdivididos en 2 subgrupos:

- Subgrupo 1 (SS): Irrigación con solución salina estéril.

- Subgrupo 2 (SU): Irrigación con solución salina estéril y activación por PUI.

Finalmente se llevó a cabo la identificación de las colonias supervivientes. El número de las mismas fue mayor $(p=0,001)$ en los subgrupos en los que no se utilizó la activación con PUI (subgrupos SS).

En 2007, Carver et al. (12) realizaron un estudio in vivo prospectivo, randomizado y doble ciego, en el que compararon la eficacia antibacteriana de una técnica manual/rotatoria frente a otra manual/rotatoria/ ultrasónica en conductos mesiales de molares mandibular necrótica de 31 pacientes adultos. Los 31 dientes se dividieron en 2 grupos: El grupo 1 estaba formado por 16 dientes preparados con instrumentación manual y rotatoria e irrigados con $\mathrm{NaOCl}$ al $6 \%$ mediante jeringa. El grupo 2 estaba compuesto por 15 dientes preparados de igual forma que el primero, pero se añadió posteriormente 1 minuto de irrigación continua ultrasónica por conducto. Se llegó a la con-

\section{TABLA 1.- CLASIFICACIÓN DE ARTÍCULOS TOTALES INCLUIDOS EN LA REVISIÓN}

\begin{tabular}{|l|c|c|c|c|}
\hline $\begin{array}{l}\text { Tipo de } \\
\text { estudio/Aspecto } \\
\text { a tratar }\end{array}$ & $\begin{array}{c}\text { Capacidad de } \\
\text { eliminación } \\
\text { de bacterias }\end{array}$ & $\begin{array}{c}\text { Capacidad de } \\
\text { eliminación } \\
\text { de detritus }\end{array}$ & $\begin{array}{c}\text { Capacidad de } \\
\text { eliminación del } \\
\text { barrillo dentinario }\end{array}$ & $\begin{array}{c}\text { Capacidad de } \\
\text { penetración del irrigante; } \\
\text { extrusión apical }\end{array}$ \\
\hline In vitro & 3 & 8 & 3 & 5 \\
Ex vivo & 4 & 13 & 6 & 1 \\
In vivo & 2 & 2 & 0 & 7 \\
Total subgrupo & 9 & 23 & 9 & 7 \\
Total artículos & \multicolumn{4}{|l}{$43^{*}$} \\
\hline * Cinco artículos del apartado 2 (capacidad de eliminación de detritus) tratan también el tema del apartado 3 (capacidad de eliminación del barrillo \\
\cline { 2 - 4 } dentinario).
\end{tabular}


clusión de que la adición de 1 minuto de irrigación ultrasónica resultó en una reducción significativa $(p=0,0006)$ del recuento de CFUs (unidades formadoras de colonias) y cultivos positivos $(p=0,0047)$.

En 2009, Townsend y Maki (13) realizaron un estudio in vitro en el que compararon la eliminación mecánica de bacterias mediante 3 sistemas de agitación y 2 de irrigación frente a la agitación ultrasónica (PUI) en un conducto de un modelo de resina. Un total de 42 modelos se dividieron en 7 grupos. El grupo control (C) con medio de cultivo de infusión de cerebro y corazón (BHI) recibió sólo irrigación convencional con jeringa. Los demás grupos inocularon con Enterococcus faecalis y se incubaron también con BHI. Los sistemas de irrigación y agitación utilizados fueron: ultrasonidos (PUI), jeringa convencional, EndoVac, EndoActivator, F-File y sistema sónico. La agitación del irrigante (agua estéril) se llevó a cabo durante 30 segundos. Las conclusiones del estudio fueron que la agitación ultrasónica fue significativamente más efectiva que la irrigación convencional y el sistema EndoVac en la eliminación bacteriana $(p<0,05)$. Sin embargo, no había diferencia estadísticamente significativa entre la agitación ultrasónica y el uso de EndoActivator, F-File y la agitación sónica, y ninguno de los sistemas eliminó por completo todas las bacterias de los modelos.

En 2010, Harrison et al. (14) investigaron la capacidad de la irrigación ultrasónica continua (CUI) para eliminar bacterias de las paredes del conducto radicular y túbulos dentinarios de dientes extraídos. Se inoculó 130 dientes unirradiculares extraídos con E. faecalis durante 4 semanas, utilizando el mismo medio de cultivo que en el estudio anterior, el BHI. Los conductos fueron asignados al azar a un grupo control $(n=25)$ o sometidos a procedimientos de limpieza y conformación de rutina $(n=105)$. Tras esto, se crearon 2 subgrupos: En el primero se aplicó irrigación ultrasónica con hipoclorito al $1 \%$ durante 1 min. $(n=35)$ o a una semana de medicación intraconducto con hidróxido de calcio $(n=35)$. Las raíces se procesaron para microscopía de luz (tinción Brown E Brenn) $(n=28)$ o microscopía electrónica de barrido $(n=7)$. El estudio concluyó que 1 minuto de irrigación ultrasónica con $\mathrm{NaOCl}$ al $1 \%$ tras la preparación de conductos rectos es un procedimiento altamente eficaz para el control bacteriano, y que se obtenían así resultados equivalentes a 1 semana de medicación intraconducto con hidróxido cálcico. Sin embargo, ninguno de los dos métodos elimina las bacterias en su totalidad.

En el mismo año, Bhuva et al. (15) realizaron un estudio in vitro para comparar la efectividad de la irrigación de $\mathrm{NaOCl}$ al $1 \%$ con PUI respecto a la misma concentración de dicho irrigante aplicado con jeringa convencional en la erradicación de biofilms de $E$. faecalis en dientes extraídos. Se cultivaron biofilms de $E$. faecalis en 48 mitades de raíces que fueron previamente seccionadas. Tras reensamblar dichas mitades, las raíces se dividieron en 4 grupos $(n=12)$ : Los dos grupos experimentales se trataron con $\mathrm{NaOCl}$ al $1 \%$ y jeringa convencional (Grupo experimental A), y $\mathrm{NaOCl}$ al $1 \%$ junto con PUI (Grupo experimental B). De los dos grupos control, en el primero se utilizó una solución salina junto con jeringa convencional (Grupo control C), mientras que el segundo grupo no recibió ningún protocolo de irrigación (Grupo control D). Se determinó que tanto la jeringa convencional como la agitación mediante PUI son igualmente efectivas, eliminando por completo los biofilms de $E$. faecalis, pero sin existir diferencias estadísticamente significativas entre ambas técnicas. Es importante señalar que en este estudio se utilizaron sólo 40 segundos de PUI frente a 2 minutos de irrigación mediante jeringa convencional.

Alves et al. (16) realizaron un estudio en 2011 para comparar la capacidad de distintos enfoques para complementar el efecto antibacteriano de la preparación químico-mecánica en conductos ovalados. Se tomaron 54 dientes extraídos (incisivos mandibulares y segundos premolares maxilares, todos unirradiculares y con un solo conducto). Los dientes se infectaron con $E$. faecalis, se prepararon utilizando instrumentación rotatoria e irrigando con $\mathrm{NaOCl}$ al $2,5 \%$ y después se sometieron a dos protocolos adicionales. En un primer grupo, se llevó a cabo un lavado final con CLX al 0,2\% junto con activación mediante PUI (que también se empleó para activar el $\mathrm{NaOCl}$ en este grupo). En un segundo grupo, los conductos recibieron conformación extra con limas Hedström. La preparación químico-mecánica y los protocolos adicionales consiguieron una reducción bacteriana altamente significativa $(p<0,001)$. Los análisis cualitativos y cuantitativos de las muestras revelaron que el efecto antibacteriano acumulativo de la irrigación final con CLXy PUI resultó efectivo $(p=0,04)$. 
Halford et al. (17) examinaron en 2012 los efectos de la emulsión de microburbujas (ME) combinado con la agitación sónica y ultrasónica, y la reducción de biofilm bacteriano en el interior de modelos de conductos radiculares. Para ello se realizaron dos experimentos. En el primero, se utilizaron imágenes a alta velocidad para caracterizar las burbujas generadas por los sistemas de agitación, y en el segundo, se utilizaron 40 conductos de dientes unirradiculares recién extraídos, inoculados con biofilms de $E$. faecalis con 7 días de crecimiento, y se agitó una solución de $\mathrm{NaOCl}$ al 5,25\% o ME de forma sónica y ultrasónica (PUI). Se formaron 6 grupos experimentales y 1 control $(n=10)$. Se realizaron cortes dentinarios a 1 y $3 \mathrm{~mm}$ del foramen apical, y se contabilizaron las CFUs. Las conclusiones informan de un efecto sinérgico de ME combinado con la agitación ultrasónica, debido a una mejora del movimiento de las burbujas y de la eficacia antibacteriana frente al biofilm.

Paiva et al. (18) evaluaron mediante un estudio in vivo la capacidad de 2 técnicas diferentes para complementar los efectos antimicrobianos del desbridamiento químico-mecánico. En el estudio participaron 31 pacientes, a cada uno de los cuales se le trató un diente unirradicular de un único conducto con periodontitis apical. Las muestras de los conductos se tomaron al inicio del estudio (grupo S1), después de la preparación con instrumental rotatorio y de irrigación con $\mathrm{NaOCl}$ al 2,5\% (grupo S2), y a continuación, después de la irrigación con $\mathrm{NaOCl}$ al 2,5\% y activación con PUI $(n=13)$ y de un lavado final con clorhexidina al $2 \%$ $(n=14)$ (grupo S3). Tras el estudio, se llegó a la conclusión de que tanto la activación con PUI como un lavado final con CLX redujeron la incidencia de resultados bacteriológicos positivos cuando se compararon con las muestras tomadas tras la instrumentación, pero no hubo diferencias estadísticamente significativas entre ambos grupos ( $p>0,05)$.

\section{Capacidad de eliminación del tejido pulpar y restos de dentina}

En 2002, Mayer et al. (19) evaluaron no sólo la capacidad de los ultrasonidos para eliminar los restos pulpares, sino también para eliminar el barrillo dentinario (este último aspecto será tratado en el siguiente apartado). De un total de 42 dientes unirradiculares de 1 solo conducto (caninos y premolares) obtenidos, se crearon 6 grupos. Todos los conductos fueron irrigados con $\mathrm{NaOCl}$ al 5,25\% y EDTA al $17 \%$. Para la activación ultrasónica del irrigante (PUI), en los grupos 2 y 5 se utilizó una lima $\mathrm{K} \mathrm{n}^{\circ} 15$ de acero, mientras que en los grupos 3 y 6 se empleó una lima flexible de NiTi con punta roma. Los grupos 1 y 4 se utilizaron como controles negativos. Aunque todos los grupos tenían significativamente mayor cantidad de barrillo y restos de tejidos a los $3 \mathrm{~mm}$ comparados con los $9 \mathrm{~mm}(\mathrm{p}<0,05)$, no se encontraron diferencias estadísticamente significativas respecto al empleo de un sistema u otro. Esto puede deberse a que en este estudio, el EDTA se dejó en el conducto antes de la activación ultrasónica del hipoclorito sódico. No se mencionó que el EDTA fuera eliminado previo a introducir el hipoclorito, lo que pudo haber causado que los autores obtuvieran dichos resultados.

Un año más tarde, Sabins et al. (20) realizaron un estudio para determinar si la irrigación sónica y ultrasónica aplicada de forma pasiva durante 30 o 60 segundos podría reducir de forma significativa la cantidad de restos en los conductos de molares maxilares tras instrumentarlos manualmente, y si de ésta manera se podría disminuir el tiempo empleado en la preparación del conducto. Se tomaron 100 conductos de molares superiores y se instrumentaron de forma manual. Los canales se dividieron aleatoriamente en 5 grupos:

- Grupo $1(n=20)$ : No recibió tratamiento.

- Grupos 2 y 3 ( $n=40)$ : Recibieron irrigación sónica durante 30 y 60 segundos respectivamente.

- Grupos 4 y 5 ( $n=40)$ : Recibieron irrigación ultrasónica (PUI) durante 30 y 60 segundos respectivamente.

El estudio demostró que ambos métodos fueron significativamente más eficaces en la limpieza de los conductos que la instrumentación por sí sola en tan sólo 30 segundos. Al realizar la comparación entre la irrigación sónica y la ultrasónica, esta última resultó ser significativamente más efectiva.

Ferreira et al. (21) evaluaron en 2004 la capacidad de limpieza de los conductos radiculares de la instrumentación rotatoria y la irrigación ultrasónica (PUI). Se utilizaron 12 incisivos mandibulares con un solo conducto y se dividieron de forma aleatoria en 3 grupos 
según el método de irrigación empleado (todas las piezas se irrigaron con $\mathrm{NaOCl}$ al $1 \%$ ):

- El grupo 1 se irrigó con $5 \mathrm{ml}$ de $\mathrm{NaOCl}$ mediante jeringa convencional entre cada instrumento.

- El grupo 2 se irrigó con $5 \mathrm{ml}$ de $\mathrm{NaOCl}$, activado durante 1 minuto con ultrasonidos entre cada lima.

- El grupo 3 se irrigó con $5 \mathrm{ml} \mathrm{NaOCl}$ con jeringa convencional entre cada lima y se realizó un lavado final activado por ultrasonidos durante 3 minutos.

El estudio mostró resultados significativamente más positivos en el grupo 3 (instrumentación rotatoria más 3 minutos de activación ultrasónica final).

Ese mismo año, Lee et al. (22) investigaron la influencia del diámetro y la conicidad de los conductos radiculares en la efectividad de la irrigación ultrasónica (PUI) para eliminar restos dentinarios colocados de forma artificial en canales radiculares artificiales secundarios no instrumentados. Se utilizaron 36 bloques de resina que se dividieron en 3 grupos $(n=12)$, según se instrumentaran a 20/.04 ProFile (grupo 1), a 20/.06 rotary GT (grupo 2) o a 20/.08 rotary GT (grupo 3). Para la irrigación se utilizó $\mathrm{NaOCl}$ al 2\% activado ultrasónicamente durante 3 minutos. Tras el estudio, los resultados mostraron que la mayor cantidad de restos de dentina se encontraron en el grupo con menor conicidad, pero no hubo diferencias estadísticamente significativas entre los grupos de mayor conicidad.

En el mismo año, dichos autores (23) realizaron otro estudio, esta vez ex vivo, para comparar la capacidad de la jeringa convencional y la irrigación ultrasónica para eliminar restos dentinarios colocados en irregulares simuladas en el interior de conductos. Se tomaron 12 caninos superiores e inferiores, que se separaron de forma longitudinal. Se creó un surco y 3 pequeños agujeros en cada conducto, en los que se colocó los restos de dentina. Todas las piezas se instrumentaron de igual forma (limas FlexoFile), irrigando con $2 \mathrm{ml}$ de $\mathrm{NaOCl}$ al $2 \%$ entre cada instrumento. La irrigación final con ultrasonidos (PUI) se realizó en 8 piezas, y en las 4 restantes se efectuó la irrigación con jeringa convencional, efectuándose el proceso 2 veces en cada diente, para obtener igual número de piezas tratadas que en el grupo de ultrasonidos, dado que con la jeringa convencional no se alteran las paredes del conducto. Tras el estudio, se concluyó que la irrigación con ultrasonidos es más efectiva que la jeringa convencional para eliminar restos de dentina en lugares no instrumentados del conducto.

En 2005, Gutarts et al. (24) compararon en un estudio in vivo la eficacia para eliminar restos de tejido de una preparación manual/rotatoria frente a otra manual/rotatoria/ultrasónica en conductos mesiales de molares mandibulares vitales. El grupo 1 estaba formado por 16 dientes preparados con una técnica manual/rotatoria, mientras que el grupo 2 estaba formado por 15 dientes preparados de igual forma pero seguidos de 1 minuto de irrigación ultrasónica por conducto (CUI). Se utilizaron 5 molares sin instrumentar como grupo control. Tras la extracción, los resultados revelaron que la utilización de 1 minuto de agitación ultrasónica tras la preparación del conducto resulta muy eficaz en la limpieza del conducto radicular.

En el mismo año, van der Sluis et al. (25) evaluaron con un estudio ex vivo la influencia de la conicidad de los conductos en la eficacia de la irrigación ultrasónica para eliminar restos dentinarios colocados artificialmente. Se tomaron 44 caninos superiores e inferiores y se dividieron en 3 grupos:

- Grupo 1 ( $n=14)$ : Se instrumentó a 20/.06 System GT.

- Grupo $2(n=14)$ : Se instrumentó a 20/.08 System GT.

- Grupo 3 ( $n=16)$ : Se instrumentó a 20/.10 System GT.

Cada conducto se dividió en 2 mitades. En una mitad se creó un surco a 2-6 mm del ápice, que se rellenó con restos de dentina. Las mitades se volvieron a unir y se recubrieron con cera. En cada canal se aplicó irrigación ultrasónica (PUI) utilizando $\mathrm{NaOCl}$ al $2 \%$. Se tomaron imágenes antes y después con un microscopio y una cámara digital. Tras el estudio se llegó a la conclusión de que la irrigación ultrasónica fue más eficaz al eliminar restos de dentina en los molares instrumentados con mayor conicidad.

Un año después, los mismos autores (26), realizaron un estudio para determinar la influencia del volumen, irrigante y método de irrigación en la eliminación de restos de dentina de la zona apical de los conductos durante la irrigación pasiva ultrasónica. Se tomaron 15 caninos, y se instrumentaron todos a 20/.10 GT. Al igual que en el estudio anterior, las piezas se separaron en 2 mitades longitudinalmente y se creó un surco en el interior. En el grupo 1, el canal se lavó con flujo continuo de $50 \mathrm{ml} \mathrm{NaOCl}$ al 2\% activa- 
do ultrasónicamente (PUI); en el grupo 2, no se usó flujo continuo sino una jeringa convencional con 12 $\mathrm{ml} \mathrm{NaOCl}$ al 2\%, a $2 \mathrm{ml} / 30$ seg.; en el grupo 3, se efectuó el mismo procedimiento que en el 2, pero con $6 \mathrm{ml}$ de $\mathrm{NaOCl}$ al $2 \%$ a $2 \mathrm{ml} / \mathrm{min}$. El grupo 4 se trató de la misma forma que el 1, pero utilizando agua como irrigante (grupo control). Dado que las puntas ultrasónicas no dañan la dentina radicular, el proceso se realizó en los mismos 15 dientes. Se tomaron imágenes antes y después de la irrigación. Como conclusión, se observó que el grupo control (agua como irrigante) no eliminó los restos de dentina de los surcos, mientras que los demás procedimientos sí. Sin embargo, no se observaron diferencias estadísticamente significativas entre los distintos grupos.

Passarinho-Neto et al. (27) evaluaron mediante un estudio in vitro la capacidad de limpieza de la instrumentación rotatoria Ni-Ti (ProFile GT) junto con la irrigación ultrasónica. Se utilizaron 36 incisivos mandibulares, que se asignaron de forma aleatoria a 4 grupos: El grupo 1 se irrigó con $100 \mathrm{ml} \mathrm{NaOCl}$ al $1 \%$ mediante jeringa convencional (grupo control); Los grupos 2, 3 y 4 se irrigaron de igual forma que el grupo anterior, pero con 1, 3 y 5 minutos de activación ultrasónica adicional (PUI). Tras la preparación, se realizó una observación histológica de las muestras. Se llegó a la conclusión de que el grupo que eliminó menor cantidad de restos fue el grupo control, mientras que el que mayor cantidad eliminó fue el grupo 4 (5 minutos de irrigación ultrasónica adicional).

Burleson et al. (28) realizaron en 2007 un estudio in vivo, prospectivo, randomizado y simple-ciego para comparar histológicamente la eficacia de eliminación de biofilm y restos de tejido de raíces mesiales de molares necróticos mediante técnica manual/rotatoria frente a otra manual/rotatoria/ultrasónica. En el estudio participaron 20 pacientes, tratándose en cada uno un molar inferior. Los dos grupos se instrumentaron de igual forma, pero en el segundo se llevó a cabo una irrigación final adicional mediante ultrasonidos (CUI) durante 1 minuto. Tras la extracción, se realizaron preparaciones histológicas y las tinciones, y se realizaron cortes transversales de $0,2 \mu \mathrm{m}$ de los últimos 1-3 $\mathrm{mm}$ apicales. Los resultados mostraron que el grupo activado mediante ultrasonidos poseía significativamente mayor capacidad de limpieza tanto en los canales como en los istmos.
Lui et al. (29) compararon mediante un estudio in vitro la eficacia de Smear Clear (EDTA al 17\% con surfactantes añadidos), con y sin el uso de ultrasonidos para la eliminación de restos de tejido y barrillo dentinario. Se extrajeron 75 dientes y se distribuyeron entre 5 grupos. Todos se trabajaron utilizando instrumentación rotatoria (Profile) y se sometieron a distintos regímenes irrigadores finales: El grupo A recibió $\mathrm{NaOCl}$ al $1 \%$; el grupo B, EDTA al $17 \%$; el grupo C, EDTA al $17 \%$ con ultrasonidos (PUI); el grupo D, Smear Clear y, el grupo E, Smear Clear con PUI. Las muestras se observaron con microscopio electrónico de barrido y se midió la eliminación de restos de tejido y de barrillo dentinario. Los análisis estadísticos mostraron que los grupos D (sólo Smear Clear) y E (Smear Clear+PUI) no aportaron resultados significativamente mejores que los grupos B y C. Se obtuvieron mejores resultados con el grupo $\mathrm{C}$ que con el $\mathrm{B}$.

Al-Jadaa et al. (30) compararon in vitro la irrigación pasiva ultrasónica (PUI) con $\mathrm{NaOCl}$ al 2,5\% con la irrigación convencional mediante administración pasiva de $\mathrm{NaOCl}$ al 2,5\% en la eliminación de los restos necróticos de tejido de canales accesorios simulados en modelos de resina epoxi. Se tomaron para el estudio 6 modelos de resina, en los que se crearon canales accesorios que fueron rellenados con tejido pulpar necrótico de bovino. Se midió la temperatura del conducto principal cada minuto, y se tomó una fotografía digital. En el grupo control, no se realizó la agitación ultrasónica, sino que se calentó el hipoclorito a la temperatura que alcanzó el grupo experimental, para imitar la temperatura conseguida. Los experimentos se repitieron 5 veces. La conclusión del estudio fue que la irrigación pasiva ultrasónica consiguió significativamente mayor disolución de tejido $(p<0,05)$.

En el mismo año, Kuah et al. (31) evaluaron la efectividad in vitro del EDTA al $17 \%$ con y sin agitación por PUI para la eliminación de restos de tejido y barrillo dentinario. Se extrajeron 105 premolares que se distribuyeron al azar en 7 grupos. Todos ellos se instrumentaron e irrigaron con diferentes técnicas de lavado final: En el grupo A se activó una solución salina con ultrasonidos durante 3 minutos; Los grupos $\mathrm{B}$ y $\mathrm{C}$, se irrigaron con $\mathrm{NaOCl}$ al $1 \%$ durante 3 minutos sin y con ultrasonidos respectivamente. Los grupos D y E se irrigaron con EDTA al 17\% durante 3 minutos sin y con ultrasonidos respectivamente. Los 
grupos F y G se irrigaron con EDTA al 17\% durante 1 minuto sin y con ultrasonidos respectivamente. Las piezas dentales se examinaron con microscopio electrónico de barrido y se evaluó la eliminación de barrillo dentinario y restos de tejido. El estudio mostró que los grupos en los que se aplicó EDTA e irrigación ultrasónica (grupos E y G) tenían significativamente mayor número de piezas con completa remoción de restos de tejido y barrillo dentinario, sin haber diferencia estadísticamente significativa entre ambos grupos. Por tanto, se concluye que la aplicación de 1 minuto de EDTA al $17 \%$ junto con activación ultrasónica es eficaz en la eliminación de restos de tejido y barrillo dentinario en la zona apical del conducto radicular.

En 2010, Klyn et al. (32) realizaron un estudio in vitro para comparar la eficacia de eliminación de restos de tejido de EndoActivator, F-file, irrigación ultrasónica (PUI) o $\mathrm{NaOCl}$ al $6 \%$ por sí solo en morales inferiores tras la instrumentación manual-rotatoria. Se utilizaron 40 molares inferiores, que se incluyeron en un bloque de resina para simular las condiciones reales. Todos recibieron el mismo procedimiento para la instrumentación rotatoria (Profile GT). Posteriormente se distribuyeron en 4 grupos experimentales $(n=10)$ según el lavado final:

- Grupo 1: Se usó el sistema F file durante 30 segundos para activar $2 \mathrm{ml}$ de $\mathrm{NaOCl}$ al $6 \%$.

- Grupo 2: Se usó EndoActivator ${ }^{\circledR}$ durante 30 segundos para activar $2 \mathrm{ml}$ de $\mathrm{NaOCl}$ al $6 \%$.

- Grupo 3: Se usó la irrigación pasiva ultrasónica (PUI) para activar $2 \mathrm{ml} \mathrm{NaOCl}$ al 6\%.

- Grupo 4: Se activaron $2 \mathrm{ml}$ de $\mathrm{NaOCl}$ al 6\% mediante una jeringa convencional.

Los resultados no mostraron diferencias estadísticamente significativas entre los distintos grupos en la limpieza de los conductos.

En el mismo año, Rödig et al. (33) realizaron un estudio ex vivo para comparar la eficacia de la jeringa convencional, RinsEndo ${ }^{\circledR}$ y la irrigación pasiva ultrasónica (PUI) para eliminar los restos de tejido de irregularidades simuladas en conductos radiculares con distintos tamaños de ápice. Se utilizaron para el estudio 30 premolares que fueron divididos de forma aleatoria entre 3 grupos $(n=10)$ y preparados con instrumentación rotatoria Flex-Master ${ }^{\circledR}$. Los dientes se separaron longitudinalmente y se creó un surco y 3 pequeñas cavidades que se rellenaron con restos de dentina. Posteriormente las partes se volvieron a ensamblar. La cantidad de restos de dentina se evaluó al microscopio con $30 \times$ de aumento. La conclusión del estudio fue que la irrigación pasiva ultrasónica es más efectiva que la jeringa convencional y el RinsEndo ${ }^{\circledR}$ en la eliminación de restos de tejido.

En el mismo año, estos autores (34) desarrollaron un estudio ex vivo para comparar la eficiencia de un sistema sónico (Vibringe), irrigación convencional e irrigación pasiva ultrasónica (PUI) en la eliminación de residuos de irregularidades simuladas en el conducto radicular. Se utilizaron para el estudio 10 incisivos laterales superiores, en los que se crearon dos surcos en las partes apical y coronal, y que se rellenaron con restos de dentina. Las conclusiones del estudio fueron que la irrigación pasiva ultrasónica es significativamente más efectiva que los otros 2 sistemas $(p<0,0001)$ y que la irrigación sónica aportó mejores resultados que la jeringa convencional.

Nuevamente, los mismos autores (35) realizaron en 2010 un estudio ex vivo para evaluar la capacidad de diferentes técnicas de agitación para eliminar los detritus y el barrillo dentinario de raíces curvas. Se tomaron raíces curvas de 108 molares mandibulares, y se prepararon con instrumentos de Ni-Ti y un lavado final de $\mathrm{NaOCl}$ y EDTA. Las muestras se asignaron a 4 grupos $(n=20)$ y se sometieron a los distintos regímenes de irrigación-agitación: No agitación (control), irrigación pasiva ultrasónica (PUI), EndoActivator y cepillo CanalBrush. Las raíces se separaron en sentido longitudinal y se observaron con el microscopio electrónico de barrido. No se encontraron diferencias estadísticamente significativas entre los distintos grupos en cuanto a la eliminación de detritus. El EndoActivator resultó más efectivo que la agitación ultrasónica y el CanalBrush.

En 2011, Adcock et al. (36) compararon en un estudio ex vivo la eficacia de la jeringa convencional y la irrigación continua ultrasónica (CUI) para eliminar restos en raíces mesiales de primeros molares inferiores con istmos estrechos utilizando un sistema cerrado de conductos. Se seleccionaron 20 piezas, las cuales se sellaron en el ápice con polinivilsiloxano y se instrumentaron con ProFile. Se crearon dos grupos: 
- Grupo $1(\mathrm{n}=10)$ : Los conductos se irrigaron con $15 \mathrm{ml}$ de $\mathrm{NaOCl}$ al $6 \%$, seguido de EDTA al $17 \%$ mediante jeringa convencional.

- Grupo $2(n=10)$ : Se realizó el mismo procedimiento que en el grupo 1 , pero activando los irrigantes mediante irrigación ultrasónica continua (CUI) durante 2 minutos.

Los análisis indicaron que no había diferencias estadísticamente significativas entre los dos métodos en cuanto a la limpieza general del conducto, pero la agitación mediante CUI sí mostró ser significativamente superior a la jeringa convencional en la limpieza de los istmos.

En el mismo año, Paqué et al. (37) realizaron un estudio ex vivo para investigar el impacto de procedimientos de irrigación secuencial en los niveles de restos de tejidos duros acumulados en el sistema de conductos de raíces mesiales de molares inferiores. Se utilizaron para el estudio 20 molares inferiores, que fueron instrumentados con el sistema ProTaper, e irrigados con $1 \mathrm{ml} \mathrm{NaOCl}$ al $1 \%$, seguidos de un lavado final con 5 $\mathrm{ml}$ de la misma solución y EDTA al 17\%. Para simular un sistema cerrado de conductos, los ápices se sellaron con cera. A continuación, se utilizó la irrigación ultrasónica pasiva (PUI). Para el análisis de las muestras se realizó un escáner $\mu \mathrm{CT}$. Los resultados mostraron que el EDTA y la irrigación con PUI producen un efecto estadísticamente significativo en la eliminación de los restos de tejido, aunque quedaron muchas zonas del conducto sin limpiar.

Howard et al. (38) realizaron ese mismo año un estudio in vitro para comparar la efectividad de la capacidad de eliminar detritus de EndoVac y PiezoFlow (CUI), y la jeringa convencional en molares mandibulares. Las muestras se irrigaron con $2 \mathrm{ml}$ de $\mathrm{NaOCl}$ al $2 \%$ y $2 \mathrm{ml}$ de EDTA al 17\%. Se tomaron imágenes de los conductos y los istmos antes de la fase de lavado final. No se encontraron diferencias estadísticamente significativas en la limpieza de los conductos e istmos entre las 3 técnicas antes y después del lavado final. Cabe señalar que, en este estudio, no se tuvo en cuenta un aspecto tan importante como es el tiempo de aplicación de cada sistema de agitación de irrigantes.

En 2012, Jiang et al. (8) compararon mediante un estudio ex vivo la eficacia de eliminación de restos de dentina de surcos artificiales creados en conduc- tos radiculares mediante seis técnicas diferentes de irrigación final. Se tomaron 20 caninos superiores que se incluyeron en resina y se separaron longitudinalmente para crear un surco longitudinal de $4 \mathrm{~mm}$ de longitud a 2-6 mm del ápice que se rellenó con restos de dentina. Las partes se volvieron a ensamblar y se instrumentaron con sistema GT. Los sistemas evaluados fueron: Irrigación convencional con jeringa, irrigación dinámica manual con gutapercha, el sistema por presión Safety Irrigator (estos tres durante 1 minuto), la irrigación ultrasónica continua (CUI) (durante 30 segundos) y un sistema por presión negativa (ANP) (durante 50 segundos). El irrigante utilizado fue $2 \mathrm{ml}$ de $\mathrm{NaOCl}$ al $6 \%$. Tras esto, se evaluó la cantidad de restos de dentina restantes. Al finalizar el estudio se concluyó que el sistema CUI fue el más efectivo de todos $(p<0,001)$, y la jeringa convencional fue el menos efectivo $(p<0,005)$.

En el mismo año, Curtis et al. (39) comparan in vitro un sistema de irrigación continua ultrasónica con la irrigación convencional con jeringa como métodos de irrigación final en la limpieza de la zona apical del conducto radicular. Se extrajeron 46 dientes (incisivos centrales superiores, laterales, caninos, incisivos centrales inferiores, laterales y primeros premolares), de los cuales se emparejaron 40. Los conductos se prepararon con instrumental rotatorio Profile a 29/.04 e irrigación con $\mathrm{NaOCl}$ al $6 \%$. Un diente de cada par se asignó al azar para recibir irrigación final con jeringa convencional o CUI. Se emplearon $5 \mathrm{ml} \mathrm{de} \mathrm{NaOCl}$ al 6\%, $5 \mathrm{ml}$ de EDTA al $15 \%$, y $5 \mathrm{ml}$ de $\mathrm{NaOCl}$ al $6 \%$. Tras el estudio se concluyó que la irrigación final con CUI en comparación con la irrigación convencional con jeringa deja significativamente menos desechos presentes en los conductos radiculares a 1 y $3 \mathrm{~mm}$ de la longitud de trabajo.

Ribeiro et al. realizaron un estudio ex vivo (40) para evaluar tanto la eliminación de restos de tejido como de barrillo dentinario por parte de distintas técnicas de irrigación. Cincuenta incisivos inferiores se dividieron en 5 grupos $(n=10)$ según la técnica utilizada: irrigación convencional con jeringa, irrigación con jeringa cubierta por cepillo NaviTip, irrigación dinámica manual, irrigación pasiva ultrasónica, y sistema de irrigación por presión negativa (EndoVac). Como solución irrigadora se utilizó $5 \mathrm{ml} \mathrm{NaOCl}$ al 2,5\% entre cada instrumento y se realizó un lavado final con EDTA al 17\% durante 1 minuto. Tras la instrumentación, las 
raíces se separaron en sentido longitudinal y se observaron con microscopio electrónico de barrido. Tras el estudio se concluyó que la irrigación dinámica manual dejó mayor cantidad de restos que los demás métodos, mientras que la irrigación ultrasónica (PUI) y EndoVac fueron los más efectivos $(p<0,05)$.

\section{Capacidad de eliminación del barrillo dentinario}

En el estudio de Mayer et al. (19) comentado en el apartado anterior, también se evaluó la eliminación del barrillo. Como se dijo anteriormente, aunque todos los grupos tenían significativamente mayor cantidad de barrillo a los $3 \mathrm{~mm}$ comparados con los $9 \mathrm{~mm}(\mathrm{p}<0,05)$, no se encontraron diferencias estadísticamente significativas respecto al empleo de un sistema u otro, lo que puede deberse al protocolo seguido en la realización del estudio.

En el año 2002, Guerisoli et al. (41) evaluaron la capacidad de diferentes soluciones irrigadoras acompañadas de 1 minuto de agitación con ultrasonidos (PUI) para la eliminación del barrillo dentinario. Se tomaron 20 incisivos mandibulares con un solo conducto y se dividieron en 4 grupos iguales. Se instrumentaron tres de los grupos. Las paredes de los conductos de los grupos 1 y 2 se cubrieron con barrillo dentinario (controles positivos). El primer grupo se irrigó con agua destilada, el segundo sólo con $\mathrm{NaOCl}$ $1 \%$ y en el tercer grupo se asoció $\mathrm{NaOCl}$ al $1 \%$ con EDTAC al $15 \%$ entre cada lima. El cuarto grupo (control negativo), que no se instrumentó, se irrigó con $\mathrm{NaOCl}$ al $1 \%$ y EDTAC $15 \%$. Finalmente, se concluyó que los conductos irrigados con $\mathrm{NaOCl}$ al $1 \%$ y EDTAC al 15\% seguido de agitación ultrasónica tenían menos barrillo dentinario $(\mathrm{p}<0,001)$, mientras que en el grupo en el que sólo se utilizó agua destilada y $\mathrm{NaOCl}$ al $1 \%$ sólo no se eliminó el barrillo. No se encontraron diferencias estadísticamente significativas entre los distintos grupos respecto a los tercios radiculares.

En el artículo de Lui et al. (29) ya comentado en el apartado anterior, compararon la eficacia de Smear Clear (EDTA al 17\% con surfactantes añadidos), con y sin el uso de ultrasonidos para la eliminación de restos de tejido y barrillo dentinario. Los análisis estadísticos mostraron que El uso de EDTA al 17\% con PUI mejoró la eliminación del barrillo dentinario, especialmente en la zona apical.
En 2008, Chopra et al. (42) compararon la efectividad de limas F ProTaper y ultrasonidos para eliminar el barrillo dentinario de conductos instrumentados cuando se irrigaron con $\mathrm{NaOCl}$ y EDTA. Sesenta premolares sanos se instrumentaron y se dividieron en 6 grupos iguales: Los grupos 1, 2 y 3 se irrigaron con un lavado final de $10 \mathrm{ml}$ de EDTA al $17 \%$ y $10 \mathrm{ml}$ de $\mathrm{NaOCl}$ al 6\%. En el grupo 1 se utilizó la lima F-file ProTaper durante 30 segundos para activar los irrigantes; en el grupo 2, se usó una lima $\mathrm{K} \mathrm{n}^{\circ} 20$ con el aparato de ultrasonidos (PUI) durante 1 minuto; en el grupo 3, se empleó únicamente la técnica convencional con jeringa. En el grupo 4 (grupo control) se utilizó una solución salina, introducida en el conducto mediante la técnica convencional con jeringa, sin lavado final. Los grupos 5 y 6 se irrigaron únicamente con 10 $\mathrm{ml} \mathrm{NaOCl}$ al $6 \%$ como lavado final, sin utilizar EDTA. En el grupo 5 el irrigante se activó mediante la lima Ffile ProTaper durante 30 segundos y el grupo 6 se activó mediante una lima $\mathrm{K} \mathrm{n}^{\circ} 20$ con el aparato de ultrasonidos (PUI) durante 1 minuto. La eliminación del barrillo dentinario se visualizó mediante microscopio electrónico de barrillo. Los tratamientos más efectivos para eliminar el barrillo dentinario fueron aquellos en los que se utilizó el EDTA, es decir, la eliminación del barrillo dentinario está principalmente influenciado por el empleo de EDTA, no por el sistema de activación.

En el estudio ya comentado en el apartado anterior de Kuah et al. (31) se evaluó la efectividad in vitro de EDTA al $17 \%$ con y sin agitación por PUI para la eliminación del barrillo dentinario. Como dijimos anteriormente, el estudio mostró que los grupos en los que se aplicó EDTA e irrigación ultrasónica (grupos E y G) tenían significativamente mayor número de piezas con completa remoción de barrillo dentinario y restos de tejido, sin haber diferencia estadísticamente significativa entre ambos grupos. Por tanto, se concluye que la aplicación de 1 minuto de EDTA al $17 \%$ junto con activación ultrasónica es eficiente y eficaz en la eliminación de barrillo dentinario y restos de tejido en la zona apical del conducto radicular.

Como ya se comentó en el apartado anterior, Rödig et al. (33) realizaron en 2010 un estudio ex vivo para evaluar la capacidad de diferentes técnicas de agitación para eliminar los detritus y barrillo dentinario en raíces curvas. En la zona coronal, la agitación de irrigantes produjo una eliminación mayor de barrillo dentinario 
que en el grupo control. El EndoActivator resultó más efectivo que la agitación ultrasónica y el CanalBrush.

En 2011, Blank-Gonçalves et al. (43) evaluaron la efectividad de diferentes técnicas agitadoras de irrigantes en la eliminación de barrillo dentinario conductos curvos. Se tomaron conductos mesiovestibulares de 62 molares inferiores y se instrumentaron con el sistema ProTaper. Las muestras se dividieron en 3 grupos de acuerdo a la técnica irrigadora final: irrigación convencional, sónica o irrigación pasiva ultrasónica. El grupo control estaba compuesto por 2 piezas que no recibieron ninguna irrigación final. En todos los grupos se utilizó $5 \mathrm{ml}$ de EDTA al 17\% durante 1 minuto y $5 \mathrm{ml}$ de $\mathrm{NaOCl}$ al 2,5\% durante 30 segundos. Como conclusión, se obtuvo que la agitación sónica y ultrasónica eliminaron significativamente más barrillo dentinario en el tercio apical que la irrigación convencional, siendo el método PUI ligeramente superior ( $80 \%$ eliminación de barrillo frente a 75\% de irrigación sónica).

Saber et al. (44) compararon la eliminación de barrillo dentinario después de la activación final del irrigante con presión negativa (ANP), agitación dinámica manual e irrigación pasiva ultrasónica (PUI). Para ello se utilizaron 40 premolares mandibulares unirradiculares que se prepararon mediante el sistema ProTaper y $\mathrm{NaOCl}$ al 2,5\%. Las muestras se dividieron en 4 grupos iguales $(n=10)$ según la técnica de irrigación final:

- Grupo 1: Irrigación pasiva.

- Grupo 2: Presión negativa.

- Grupo 3: Agitación dinámica manual.

- Grupo 4: Irrigación pasiva ultrasónica (PUI).

Tras el estudio se concluyó que la activación final con presión negativa y la agitación manual eliminaron más barrillo dentinario que la irrigación pasiva y PUI. Esta falta de eficacia en la eliminación del barrillo por parte de los ultrasonidos, probablemente se deba al pequeño tamaño de la punta ultrasónica utilizada y a que la potencia empleada durante la aplicación de este sistema fue demasiado débil.

En el estudio de Ribeiro et al. (40) comentado anteriormente, se evaluó tanto la eliminación de restos de tejido como de barrillo dentinario por parte de distintas técnicas de irrigación. Tras el estudio se concluyó que respecto a la eliminación del barrillo, no hubo diferencia estadísticamente significativa entre grupos (a pesar de que las imágenes obtenidas mostraran túbulos mucho más abiertos en el grupo irrigado con P(II).

\section{Capacidad de penetración de la solución irrigadora: En conductos laterales, túbulos dentinarios y a la longitud de trabajo. Seguridad de la irrigación ultrasónica: Extrusión apical}

Desai y Himel (45) evaluaron en 2009 la seguridad de varios sistemas de irrigación intraconducto midiendo la extrusión apical del irrigante. Para el estudio se tomaron 22 dientes unirradiculares, que fueron instrumentados y asegurados a través de la tapa de un vial de centelleo para recoger todo el irrigante extruído apicalmente. Los sistemas de irrigación utilizados fueron EndoVac, EndoActivator, irrigación dinámica manual con jeringa convencional, irrigación ultrasónica continua (CUI) y RinsEndo. Todos los irrigantes se probaron en los mismos 22 dientes para evitar diferencias en la anatomía de conductos y diámetro apical. Como conclusión, el sistema EndoVac no produjo extrusión, y el EndoActivator causó menor extrusión que los grupos de irrigación manual, ultrasónica y sónica de manera estadísticamente significativa. El problema principal respecto al protocolo de este estudio (y que reconocen los propios autores) es que no se simulan las condiciones normales de la clínica, en las que el tejido periodontal y el hueso proporcionan resistencia a la extrusión apical, debido al fenómeno de vapor lock.

En un estudio realizado por Gregorio et al. (46) se evaluó la penetración del hipoclorito de sodio al $5,25 \%$ sólo y en combinación con EDTA al $17 \%$ en conductos laterales simulados mediante activación sónica y ultrasónica. Se crearon 480 conductos laterales en 80 dientes extraídos y posteriormente diafanizados, mediante limas K 06 a 2, 4,5 y 6 mm de la longitud de trabajo. Las muestras se rodearon de silicona transparente para simular la presencia circundante de los tejidos periodontales. Las piezas se asignaron de forma aleatoria a 4 grupos:

- Grupo $1(n=20)$ : Irrigado con $\mathrm{NaOCl}$ al 5,25\% activado sónicamente.

- Grupo $2(n=20)$ : Irrigado con $\mathrm{NaOCl}$ al 5,25\% activado ultrasónicamente.

- Grupo $3(n=20)$ : Irrigado con $\mathrm{NaOCl}$ al 5,25\% más EDTA al $17 \%$ activados sónicamente.

- Grupo $4(n=20)$ : Irrigado con $\mathrm{NaOCl}$ al 5,25\% más EDTA al $17 \%$ activados ultrasónicamente. 
De la realización del estudio se concluyó que tanto la activación sónica como la ultrasónica ofrecieron una mejor irrigación de los conductos laterales a 2 y 4,5 mm de la longitud de trabajo en comparación con la irrigación convencional. No se observó diferencia estadísticamente significativa entre la irrigación sónica y la ultrasónica.

En 2010, los mismos autores (47) evaluaron el efecto de distintos sistemas de irrigación y activación en la penetración del hipoclorito de sodio en conductos laterales simulados y a la longitud de trabajo en un sistema cerrado. Se utilizaron 100 dientes unirradiculares, creándose un total de 600 canales laterales. Para simular la situación clínica real, se creó un sistema cerrado cubriendo cada raíz con cera de verano. Las raíces se asignaron aleatoriamente a 4 grupos experimentales $(n=20)$ :

- El grupo 1 recibió activación sónica.

- El grupo 2 recibió activación por PUI.

- El grupo 3 recibió activación mediante sistema F-file (lima de plástico de superficie lisa).

- El grupo 4 recibió irrigación mediante un sistema de presión negativa y, por último,

- El grupo 5 recibió irrigación mediante un sistema de presión positiva (grupo control).

Las muestras se evaluaron de igual forma al trabajo anterior. Los resultados mostraron que el grupo que recibió la irrigación por presión negativa fue el que más se acercó a la longitud de trabajo, mientras que el grupo activado mediante el sistema PUI fue el más efectivo en la penetración de canales laterales.

En el mismo año, Paragliola et al. (48) examinaron el efecto de distintos protocolos de agitación de irrigantes en la penetración en los túbulos dentinarios. Se prepararon 56 dientes unirradiculares con instrumentos de NiTi, y un lavado final con $\mathrm{NaOCl} 5 \%$ marcado con alizarina roja $0,2 \%$. Para imitar la situación real, la zona exterior del tercio apical se cubrió con cera para evitar la salida del irrigante a través del foramen. Las piezas se asignaron a 7 grupos $(n=8)$ y se sometieron a los distintos protocolos de activación de irrigantes:

- Grupo 1 (control): No recibió agitación del irrigante.

- Grupo 2: Agitación con sistema K-file.

- Grupo 3: Agitación dinámica manual con gutapercha.

- Grupo 4: Agitación sónica con Plastic Endo.
- Grupo 5: Agitación sónica con EndoActivator.

- Grupo 6: Agitación ultrasónica con Satelec (PUI).

- Grupo 7: Agitación ultrasónica con EMS (PUI).

Las piezas se seccionaron a 1, 3 y 5 mm del ápex y se prepararon para microscopía por fluorescencia. Los grupos que aportaron mejores resultados fueron aquellos cuyo irrigante fue agitado mediante ultrasonidos, sin haber diferencia entre ambos. A $1 \mathrm{~mm}$ del ápice, el mejor resultado se obtuvo en el grupo EMS. Por tanto, los resultados apoyan el uso de agitación ultrasónica para aumentar la efectividad del lavado final en las paredes del conducto en el tercio apical.

En 2011, Mitchell et al. (49) compararon distintos sistemas de irrigación en 10 pares de dientes unirradiculares emparejados, que fueron instrumentados. Se midió la frecuencia y el grado de extrusión apical del hipoclorito de sodio en un ambiente periapical simulado. Se crearon dos grupos principales, según los dientes fueron instrumentados a un diámetro de 35,06 o 50,06. Cada diente se incluyó en un recipiente de plástico transparente, relleno con un gel que contiene el colorante sensible al $\mathrm{pH}$ M-cresol púrpura, que cambia de amarillo a un $\mathrm{pH}$ de 7,4 a púrpura a un $\mathrm{pH}$ de 9 . Los conductos se irrigaron con $\mathrm{NaOCl}$ al $6 \%$ mediante EndoActivator, EndoVac, Rispi-Sonic/MicroMega 1500, irrigación ultrasónica (PUI; Irrisafe) y jeringa convencional, de modo que cada diente se sometió a todos los procedimientos de irrigación en un diseño cruzado aleatorio. Como resultado del estudio, se observó que la frecuencia de extrusión fue menor en los dientes con el tamaño de la preparación apical menor (36\%) $(p=0,014)$ en el que la extrusión dependió según el sistema de irrigación utilizado, al contrario de lo que ocurrió con los dientes con la preparación apical de 50,06. En el grupo de 35,06, la frecuencia de extrusión fue menor en la irrigación con EndoVac. El sistema PUI se situó en un grado intermedio tanto en la frecuencia como en el grado de extrusión.

Un año más tarde, Castelo-Baz et al. (50) compararon mediante un estudio in vitro del efecto de dos técnicas de irrigación ultrasónica en la penetración del hipoclorito de sodio en el conducto principal y conductos laterales simulados de dientes extraídos. Se tomó un total de 60 dientes unirradiculares, en cada uno de los cuales se creó un par de conductos laterales a 2, 4 y $6 \mathrm{~mm}$ de la longitud de trabajo (6 canales por conducto, $n=360$ ). Para simular la si- 
tuación clínica, se creó un sistema cerrado en cada diente. Los dientes se asignaron de forma aleatoria a 3 grupos $(n=20)$ :

- Grupo 1: Irrigación por presión positiva.

- Grupo 2: Irrigación ultrasónica pasiva (PUI).

- Grupo 3: Irrigación ultrasónica continua (CUI).

Las muestras se evaluaron mediante observación directa de imágenes obtenidas con microscopio. Para examinar la penetración de las soluciones irrigadoras se le añadió tinta china al $20 \%$ a una solución de $\mathrm{NaOCl}$ al 5\%. Los resultados mostraron una penetración significativamente mayor $(\mathrm{p}<0,05)$ de los irrigantes en los conductos laterales en el grupo irrigado con CUI. No hubo diferencia estadísticamente significativa entre los sistemas PUI y CUI en cuanto a la penetración en los tercios apicales de los conductos principales.

Muñoz y Camacho-Cuadra (51) compararon in vivo la eficacia de la irrigación convencional, la irrigación pasiva ultrasónica (PUI), y un sistema de presión negativa para la dispensación del irrigante a la longitud de trabajo en raíces mesiales de molares mandibulares. Se tomaron 30 raíces mesiales de 30 molares mandibulares, que fueron aleatoriamente distribuidas en 3 grupos $(n=10)$ :

- Grupo 1: Jeringa convencional.

- Grupo 2: Irrisafe (PUI).

- Grupo 3: Sistema EndoVac.

Todos los conductos se irrigaron con $\mathrm{NaOCl}$ al 5,25\%. Antes de la obturación, los canales se irrigaron con 1 ml de solución radiopaca con el correspondiente sistema de irrigación, y se tomó una radiografía periapical. A pesar de que los datos resultados eran mejores en el grupo activado ultrasónicamente, no hubo diferencia estadísticamente significativa entre éste y el grupo activado mediante EndoVac, por lo que ambos son igualmente más efectivos que la irrigación convencional en cuanto a la dispensación del irrigante a la longitud de trabajo de los conductos radiculares.

\section{DISCUSIÓN}

Respecto a la capacidad de los ultrasonidos para la erradicación de bacterias, en la mayoría de los estudios analizados se empleó el sistema PUI, y se observó que su empleo después de la instrumentación provoca una reducción significativa del número de bacterias, alcanzando resultados mucho mejores que con la jeringa clásica y otros métodos de activación de irrigantes. Este sistema también causó una reducción considerable en la cantidad de unidades formadoras de colonias (CFU). Estos resultados positivos podrían atribuirse a dos factores principales: la potencia ultrasónica provoca la separación de los biofilms de la pared del conducto, y que la bacteria se hace más permeable al hipoclorito sódico gracias a un debilitamiento temporal de la membrana. Por otro lado, si bien la mayoría de los estudios avalan la eficacia de este método, un único artículo de los 9 analizados afirma que la agitación con PUI resultó igual de efectiva que la jeringa convencional (15) pero, como se dijo al comentar dicho estudio, sólo se aplicó la irrigación ultrasónica durante 40 segundos, frente a los 2 minutos durante los que se irrigó con la jeringa convencional en otro grupo. A pesar de la importante efectividad de los ultrasonidos para eliminar las bacterias, algunos reivindican que la erradicación de las mismas de las paredes del conducto no es completa $(11,13,14)$.

En cuanto a la capacidad de los ultrasonidos para eliminar el tejido pulpar y los restos de dentina, en la mayoría de los estudios analizados se empleó la irrigación ultrasónica pasiva. Hay un consenso general de que la irrigación con PUI es más efectiva en la eliminación de detritus que la jeringa convencional, lo que puede deberse a la mayor velocidad y volumen de irrigante. Además, la oscilación de la lima puede provocar que los irrigantes lleguen a zonas poco accesibles así como tener una mayor capacidad para eliminar una mayor cantidad de detritus. El empleo de CUI también avala estos resultados, especialmente en la limpieza de los istmos (36).

Respecto a la capacidad de los ultrasonidos para eliminar el barrillo dentinario, en todos los estudios analizados se empleó el sistema PUI. Hay un consenso general de que la irrigación con PUI es más efectiva en la eliminación del barrillo dentinario que cuando esta técnica de activación no se utiliza, y que es ligeramente superior a otros métodos de agitación de irrigantes. Sin embargo, en uno de los estudios se dice que es el irrigante (normalmente el EDTA) el que realmente ejerce la eliminación del barrillo dentinario, y que la acción del PUI es potenciadora de la actividad quelante (42). En uno de los artículos en los que no se aprecian buenos resultados en la eliminación del barrillo por parte de los ultrasonidos, 
se afirma que dicho hallazgo se debe al empleo de una punta ultrasónica pequeña y a una potencia demasiado baja para dicho tamaño (44).

En cuanto a la seguridad de la irrigación ultrasónica (extrusión apical), en la mayoría de los estudios analizados se empleó el sistema PUI. En ellos, se llegó a la conclusión de que la irrigación ultrasónica produce extrusión del irrigante, lo cual puede deberse en gran medida a que no se crearon unas condiciones que simulen a la perfección la situación real clínica. Por otra parte, en cuanto a la penetración de los conductos laterales, túbulos dentinarios y el alcance del irrigante a la longitud de trabajo, la mayoría de los estudios afirman que la irrigación ultrasónica es eficaz a la hora de penetrar en los conductos laterales y túbulos dentinarios, pero no hay evidencias claras sobre la capacidad del irrigante para llegar a la longitud de trabajo (de modo que contradicen los estudios en los que se afirma que incluso hay extrusión apical). Por tanto, no hay una demostración clara de si el irrigante activado por PUI llega realmente a la longitud de trabajo, probablemente por la dificultad de simular las condiciones reales.

\section{CONCLUSIONES}

La activación ultrasónica de las soluciones irrigadoras (en especial, mediante PUI), es una manera eficaz de eliminar las bacterias, los detritus y el barrillo dentinario del sistema de conductos, de manera superior a la irrigación convencional con jeringa e igual o incluso superior a otros métodos mecanizados. Además, estos sistemas ultrasónicos permiten a las soluciones irrigadoras penetrar en los conductos laterales y túbulos dentinarios de forma eficaz, de manera superior a la irrigación convencional con jeringa e igual o incluso superior a otros métodos mecanizados. Respecto a si facilita el alcance del irrigante a la longitud de trabajo o si provoca extrusión apical del mismo no hay evidencias claras, debido a la dificultad de crear un sistema que emule con total precisión la situación real.

\section{BIBLIOGRAFÍA}

1. Haapasalo M, Shen Y, Qian W, Gao Y. Irrigation in endodontics. Dent Clin North Am 2010;54: 291-312.
2. Peters OA, Schönenberger K, Laib A. Effects of four ni-ti preparation techniques on root canal geometry assessed by micro computed tomography. Int Endod J 2001;34:221-30.

3. Wu M, Wesselink PR. A primary observation on the preparation and obturation of oval canals. Int Endod J 2001;34:137-41.

4. Senia ES, Marshall FJ, Rosen S. The solvent action of sodium hypochlorite on pulp tissue of extracted teeth. Oral Surgery, Oral Medicine, Oral Pathology. 1971;31:96-103.

5. Tay FR, Gu L, Schoeffel GJ, Wi mmer C, Susin L, Zhang K, et al. Effect of vapor lock on root canal debridement by using a side-vented needle for positive-pressure irrigant delivery. J Endod 2010; 36:745-50.

6. Plotino G, Pameijer CH, Maria Grande N, So mma F. Ultrasonics in endodontics: A review of the literature. J Endod 2007;33:81-95.

7. Gu L, Kim JR, Ling J, Choi KK, Pashley DH, Tay FR. Review of contemporary irrigant agitation techniques and devices. J Endod 2009;35:791-804.

8. Jiang L, Lak B, Eijsvogels LM, Wesselink P, van der Sluis LWM. Comparison of the cleaning efficacy of different final irrigation techniques. J Endod 2012;38:838-41.

9. Van Der Sluis LWM, Gambarini G, Wu MK, Wesselink PR. The influence of volume, type of irrigant and flushing method on removing artificially placed dentine debris from the apical root canal during passive ultrasonic irrigation. Int Endod J 2006;39:472-6.

10. Weber CD, McClanahan SB, Miller GA, Diener-West $M$, Johnson JD. The effect of passive ultrasonic activation of $2 \%$ chlorhexidine or $5.25 \%$ sodium hypochlorite irrigant on residual antimicrobial activity in root canals. J Endod 2003; 29:562-4.

11. Spoleti P, Siragusa M, Spoleti MJ. Bacteriological evaluation of passive ultrasonic activation. J Endod 2003;29:12-4.

12. Carver K, Nusstein J, Reader A, Beck M. In vivo antibacterial efficacy of ultrasound after hand and rotary instrumentation in human mandibular molars. J Endod 2007;33:1038-43.

13. Townsend C, Maki J. An in vitro comparison of new irrigation and agitation techniques to 
ultrasonic agitation in removing bacteria from a simulated root canal. J Endod 2009;35:1040-3.

14. Harrison AJ, Chivatxaranukul P, Parashos P, Messer $\mathrm{HH}$. The effect of ultrasonically activated irrigation on reduction of enterococcus faecalis in experimentally infected root canals. Int Endod J 2010;43:968-77.

15. Bhuva B, Patel S, Wilson R, Niazi S, Beighton D, Mannocci F. The effectiveness of passive ultrasonic irrigation on intraradicular enterococcus faecalis biofilms in extracted single-rooted human teeth. Int Endod J 2010;43:241-50.

16. Alves FRF, Almeida BM, Neves MAS, Moreno JO, Rôças IN, Siqueira Jr. JF. Disinfecting ovalshaped root canals: Effectiveness of different supplementary approaches. J Endod 2011;37: 496-501.

17. Halford A, Ohl C, Azarpazhooh A, Basrani B, Friedman S, Kishen A. Synergistic effect of microbubble emulsion and sonic or ultrasonic agitation on endodontic biofilm in Vitro. J Endod 2012;38:1530-4.

18. Paiva SSM, Siqueira Jr. JF, Rôças IN, Carmo FL, Ferreira DC, Curvelo JAR, et al. Supplementing the antimicrobial effects of chemomechanical debridement with either passive ultrasonic irrigation or a final rinse with chlorhexidine: A clinical study. J Endod 2012;38:1202-6.

19. Mayer BE, Peters OA, Barbakow F. Effects of rotary instruments and ultrasonic irrigation on debris and smear layer scores: A scanning electron microscopic study. Int Endod J 2002;35: 582-9.

20. Sabins RA, Johnson JD, Hellstein JW. A comparison of the cleaning efficacy of short-term sonic and ultrasonic passive irrigation after hand instrumentation in molar root canals. J Endod 2003;29:674-8.

21. Ferreira RB, Alfredo E, De Arruda MP, Sousa YTCS, Sousa-Neto MD. Histological analysis of the cleaning capacity of nickel-titanium rotary instrumentation with ultrasonic irrigation in root canals. Australian Endodontic Journal 2004;30: 56-8.

22. Lee SJ, Wu MK, Wesselink PR. The effectiveness of syringe irrigation and ultrasonics to remove debris from simulated irregularities within prepared root canal walls. Int Endod J 2004;37:672-8.
23. Lee SJ, Wu MK, Wesselink PR. The efficacy of ultrasonic irrigation to remove artificially placed dentine debris from different-sized simulated plastic root canals. Int Endod J 2004;37:60712.

24. Gutarts R, Nusstein J, Reader A, Beck M. In vivo debridement efficacy of ultrasonic irrigation following hand-rotary instrumentation in human mandibular molars. J Endod 2005;31:166-70.

25. Van Der Sluis LWM, Wu M, Wesselink PR. The efficacy of ultrasonic irrigation to remove artificially placed dentine debris from human root canals prepared using instruments of varying taper. Int Endod J 2005;38:764-8.

26. Van Der Sluis LWM, Gambarini G, Wu MK, Wesselink PR. The influence of volume, type of irrigant and flushing method on removing artificially placed dentine debris from the apical root canal during passive ultrasonic irrigation. Int Endod J 2006;39:472-6.

27. Passarinho-Neto JG, Marchesan MA, Ferreira RB, Silva RG, Silva-Sousa YTC, Sousa-Neto MD. In vitro evaluation of endodontic debris removal as obtained by rotary instrumentation coupled with ultrasonic irrigation. Australian Endodontic Journal. 2006;32:123-8.

28. Burleson A, Nusstein J, Reader A, Beck M. The in vivo evaluation of Hand/Rotary/Ultrasound instrumentation in necrotic, human mandibular molars. J Endod 2007;33:782-7.

29. Lui J, Kuah H, Chen N. Effect of EDTA with and without surfactants or ultrasonics on removal of smear layer. J Endod 2007;33:472-5.

30. Al-Jadaa A, Paqué F, Attin T, Zehnder M. Necrotic pulp tissue dissolution by passive ultrasonic irrigation in simulated accessory canals: Impact of canal location and angulation. Int Endod J 2009;42:59-65.

31. Kuah H, Lui J, Tseng PSK, Chen N. The effect of EDTA with and without ultrasonics on removal of the smear layer. J Endod 2009;35:393-6.

32. Klyn SL, Kirkpatrick TC, Rutledge RE. In vitro comparisons of debris removal of the Endo ActivatorTM system, the F FileTM, ultrasonic irrigation, and $\mathrm{NaOCl}$ irrigation alone after handrotary instrumentation in human mandibular molars. J Endod 2010;36:1367-71. 
33. Rödig T, Döllmann S, Konietschke F, Drebenstedt $S$, Hülsmann $M$. Effectiveness of different irrigant agitation techniques on debris and smear layer removal in curved root canals: A scanning electron microscopy study. J Endod 2010;36:1983-7.

34. Rödig T, Hirschleb $M$, Zapf A, Hülsmann $M$. Comparison of ultrasonic irrigation and RinsEndo for the removal of calcium hydroxide and ledermix paste from root canals. Int Endod J 2011;44: 1155-61.

35. Rödig T, Sedghi M, Konietschke F, Lange K, Ziebolz $D$, Hülsmann $M$. Efficacy of syringe irrigation, RinsEndo ${ }^{\circledR}$ and passive ultrasonic irrigation in removing debris from irregularities in root canals with different apical sizes. Int Endod J 2010;43:581-9.

36. Adcock JM, Sidow SJ, Looney SW, Liu Y, McNally $\mathrm{K}$, Lindsey $\mathrm{K}$, et al.. Histologic evaluation of canal and isthmus debridement efficacies of two different irrigant delivery techniques in a closed system. J Endod 2011;37:544-8.

37. Paqué F, Boessler C, Zehnder M. Accumulated hard tissue debris levels in mesial roots of mandibular molars after sequential irrigation steps. Int Endod J 2011;44:148-53.

38. Howard RK, Kirkpatrick TC, Rutledge RE, Yaccino JM. Comparison of debris removal with three different irrigation techniques. J Endod 2011;37: 1301-5.

39. Curtis TO, Sedgley CM. Comparison of a continuous ultrasonic irrigation device and Conventional needle irrigation in the removal of root canal debris. J Endod 2012;38:1261-4.

40. Ribeiro EM, Silva-Sousa YTC, Souza-Gabriel AE, Sousa-Neto MD, Lorencetti KT, Silva SRC. Debris and smear removal in flattened root canals after use of different irrigant agitation protocols. Microsc Res Tech. 2012;75:781-90.

41. Guerisoli DMZ, Marchesan MA, Walmsley AD, Lu mley PJ, Pecora JD. Evaluation of smear layer removal by EDTAC and sodium hypochlorite with ultrasonic agitation. Int Endod J 2002;35:41821.

42. Chopra S, Murray PE, Namerow KN. A scanning electron microscopic evaluation of the effectiveness of the F-file versus ultrasonic activation of a K-file to remove smear layer. J Endod 2008;34:1243-5.
43. Blank-Gonçalves LM, Nabeshima CK, Martins GHR, MacHado MEDL. Qualitative analysis of the removal of the smear layer in the apical third of curved roots: Conventional irrigation versus activation systems. J Endod 2011;37:1268-71.

44. Saber SE, Hashem AAR. Efficacy of different final irrigation activation techniques on smear layer removal. J Endod 2011;37:1272-5.

45. Desai P, Himel V. Comparative safety of various intracanal irrigation systems. J Endod 2009;35: 545-9.

46. de Gregorio C, Estévez R, Cisneros R, Heilborn C, Cohenca N. Effect of EDTA, sonic, and ultrasonic activation on the penetration of sodium hypochlorite into simulated lateral canals: An in vitro study. J Endod 2009;35:891-5.

47. de Gregorio C, Estévez R, Cisneros R, Paranjpe A, Cohenca N. Efficacy of different irrigation and activation systems on the penetration of sodium hypochlorite into simulated lateral canals and up to working length: An in vitro study. J Endod 2010; 36:1216-21.

48. Paragliola R, Franco V, Fabiani C, Mazzoni A, Nato F, Tay FR, et al. Final rinse optimization: Influence of different agitation protocols. J Endod 2010; 36:282-5.

49. Mitchell RP, Baumgartner JC, Sedgley CM. Apical extrusion of sodium hypochlorite using different root canal irrigation systems. J Endod 2011;37:1677-81.

50. Castelo-Baz P, Martín-Biedma B, Cantatore G, Ruíz-Piñón M, Bahillo J, Rivas-Mundiña B, et al. In Vitro comparison of passive and continuous ultrasonic irrigation in simulated lateral canals of extracted teeth. J Endod 2012;38:688-91.

51. Muñoz HR, Camacho-Cuadra K. In Vivo efficacy of three different endodontic irrigation systems for irrigant delivery to working length of mesial canals of mandibular molars. J Endod 2012;38:445-8.

\section{CORRESPONDENCIA}

Juan José Segura Egea

Dpto. de Estomatología. Facultad de Odontología

Avicena, s/n

41009-Sevilla (España)

Correo electrónico: segurajj@us.es 